\title{
New times at the journal of clinical monitoring and computing
}

\author{
Stephen E. Rees ${ }^{1}$
}

Received: 10 June 2015/ Accepted: 10 June 2015/Published online: 14 June 2015

(C) Springer Science+Business Media New York 2015

I am pleased to announce that two new Associate editors have been appointed to the Journal of Clinical Monitoring and Computing, these being Professors Thomas Scheeren, from Groningen, the Netherlands and Karim Bendjelid from Geneva, Switzerland. Both Professors are well known for their clinical and technological contributions to the community and the journal, in particular in relation to hemodynamic monitoring, which remains a core area of the journal's activities.

The appointment of Professors Scheeren and Bendjelid reflects the expansion of the journal over recent years. Since 2012, we have re-established an official ISI impact factor, with this rising from 0.887 in 2012 to 1.448 in 2014. The number of submissions has doubled from 2011 to 2014 , and the number of pages has expanded from 80 to 100 per issue, with a further expansion to 120 pages planned for 2016. I believe that the increasing impact factor reflects the quality of papers published and I would like to thank all authors, reviewers and editorial board members for their continuing efforts in improving the journal.

I believe that the Journal of Clinical Monitoring and Computing is a unique journal, a technological journal dedicated to the specialist clinical fields of anesthesia, intensive care, surgical monitoring and related disciplines. Unlike most biomedical engineering journals this tight clinical focus ensures continuity of relevance to the same clinical readers. Unlike most clinical journals, its technical focus allows publication of new ideas and technology,

Stephen E. Rees

sr@hst.aau.dk

1 Respiratory and Critical Care group (rcare), Aalborg University, Aalborg, Denmark evaluated on small groups of patients. This promotes the novel solution, not yet evaluated by large randomized control trials. It is our aim to publish work which is always both technically correct and clinically relevant, and our editorial board includes a large number of anesthetists, intensivists and biomedical engineers.

Since 2011 the editorial board has been revised substantially, and organized into relevant clinical disciplines within the scope of the journal. In addition, a number of specialist society members have been added to the board. These include board members representing the European Society for Computing and Technology in Anaesthesia and Intensive Care (ESCTAIC), the Society for Technology in Anesthesia (STA), the Society for Complex Acute Illness (SCAI) and the NAVAt (NAVigating towards your Anestheisa Targets) group. These societies represent important contributions to the development of technological and mathematical solutions to clinical problems in our field, and it is a pleasure to have representation on the editorial board of the JCMC. I believe that our editorial board should be renewed regularly, and I welcome proposals from those of you who would like to contribute. Please feel free to recommend yourself or a colleague by sending an email to me including $\mathrm{cv}$ and publication list. Other suggestions, including special issues or collaboration with societies are also welcome.

Finally, in accordance with the publisher, the editorial board will select a featured article for each issue, with this being available free of charge on the journal's web page.

I look forward to our further collaboration. 